\title{
Key Considerations for Power Management in Active Implantable Medical Devices
}

\author{
Dorian Haci ${ }^{\dagger}$, Yan Liu ${ }^{\dagger}$, Sara S. Ghoreishizadeh ${ }^{\star}$, Timothy G. Constandinou ${ }^{\dagger}$ \\ $\dagger$ Centre for Bio-Inspired Technology, Department of Electrical and Electronic Eng., Imperial College London, UK \\ $\star$ Aspire Centre for Rehabilitation and Assistive Technology, University College London, UK \\ Email: \{d.haci14, yan.liu06, t.constandinou\}@imperial.ac.uk, s.ghoreishizadeh@ucl.ac.uk
}

\begin{abstract}
Within the rapidly advancing field of active implantable medical devices, power management is a major consideration. Devices that provide life critical (or avoiding life threatening) function require a dependable, always-on power source, for example pacemakers. There is then a trade-off with battery lifetime as to whether such devices employ a primary cell or rechargeable battery. With new applications requiring multi-module implants, there is now also a need for transmitting within the body from one device to another. This paper outlines the key considerations and the process to define and optimise the power management strategy. We then apply this to a case study application - developing an implanted, multi-module closed-loop neuromodulation device for the treatment of focal epilepsy.
\end{abstract}

\section{INTRODUCTION}

The need for new medical treatments, supported by recent advances in microsystem technologies, has paved the way to the development of a wide range of active implantable medical devices (AIMDs). Besides established AIMDs in the cardiovascular domain, neuromodulation devices are demonstrating a significant impact in the treatment of severe neurological disorders. These include movement disorders (e.g. Parkinson's), chronic pain and epilepsy.

Depending on their specific application, AIMDs differ in size, form factor, packaging, stimulation, recording, signal processing and communication capabilities, etc. These tightly intertwined parameters coupled with end-user desirability, business viability and healthcare constraints pose a significant challenge in developing an efficient and effective power management strategy. For example, for AIMDs that provide life critical (or avoid life threatening) function, an implantable battery is essential to guarantee an "always-on" energy source. On the other hand, AIMDs that address applications that are deemed to be low risk in the event of interrupted power supply (e.g. cochlear implants), can justify using external battery with wireless power transfer (WPT). Here, the location and/or proximity to the skin surface of the AIMD may add constraints to the feasibility of effective WPT. There is also a tradeoff between device size (i.e. battery capacity) and time to replacement surgery that may motivate the selection between a primary cell or a lower density but rechargeable battery.

With the advent of multi-module implantable devices, further considerations are needed as to how each module is powered and the energy is transmitted. Such systems typically consist of a master device (containing implantable battery) that transmits power to the other nodes (i.e. slave devices) in the system, using implantable cables. An alternative is to employ some form of in-body WPT, but this brings its own set of challenges and thus currently remains a research topic.

This paper outlines the key considerations in establishing an appropriate power management strategy during the design of an AIMD. We identify key features from the current stateof-the-art (based on example commercially available AIMDs) and identify a design process. We highlight the need for a holistic approach that evaluates power requirements from a system level and optimises the strategy with respect to target application. We then use this methodology to develop a novel power management strategy for a case study application, an AIMD to provide a closed-loop therapy for focal epilepsy.

\section{Key Considerations For Power Management}

The European Directive 90/385/EEC on AIMDs, defines an active medical device as "any medical device relying for its functioning on a source of electrical energy or any source of power other than that directly generated by the human body or gravity" [1]. Thus, when considering an implanted electronic system, such a power source needs to be established. The choice of modality used to generate, store, and distribute this energy within the implantable system depends on three main factors: (i) external requirements (e.g. end user desirability) relating to the medical application; (ii) essential application specific functions; and (iii) implementation constraints. The power management strategy should thus take into account all such key factors that are often conflicting and optimise the overall system power consumption.

\section{A. Medical Application and Energy Supply}

Most currently available AIMDs are intended for therapeutic applications. In some cases, they provide a first-line treatment for patients with life threatening conditions, such as the implantable cardioverter-defibrillator (ICD) employed for people at risk for sudden cardiac arrest, or the artificial cardiac pacemaker regulating the electrical conduction of the heart through timed pulses. Due to their life critical function, these devices must include a long lasting battery and power management circuitry to ensure the therapy is autonomous and uninterrupted.

For AIMDs that are, however, correcting or restoring 'non vital' functions, it is not critical to ensure a continuous power supply (i.e. any interruption would not risk for patients' life). An example is DBS for movement disorders such as Parkinson's disease. Although this still uses a subcutaneous chest implant with primary cell, the neuromodulation that is delivered to deep brain structures, can be enabled by way of an external programmer. This allows patients and clinicians to activate the treatment when needed. On the other hand, cochlear implants do not include an implanted battery; but rather receive their energy (and data) via an inductive transcutaneous link to an external device. It is this external device (e.g. wearable, behind the ear) that houses the battery (and thus user changeable) together with microphone, auditory 
processor and inductive coil. If this is thus detached from the scalp, the implant is turned off and the function is interrupted.

It is the application constraints that guide the choice of which energy generation modality are needed and where. This in turn defines the high-level power management architecture that is required. In battery powered implants, for example, DC/DC supply conversion is required to generate the appropriate voltage levels for the underlying circuitry; whilst in wirelessly powered implants, $\mathrm{AC} / \mathrm{DC}$ conversion is required (i.e. rectification, smoothing, regulation) to recover a stable, constant DC supply.

\section{B. Functional Requirements and Implant Lifespan}

Medical implants can interact with the human body by (i) modulating neural activity using electrical, optical, or ultrasound stimulation, and/or by (ii) sensing neural activity typically electrically. Most of the early AIMD were electrical stimulation only devices. In addition to previous examples given, neuromodulation devices such as Vagus Nerve Stimulators (VNS) for epilepsy and Spinal Cord Stimulators (SCS) for chronic pain also belong to this category, as they provide open loop therapies. The power consumption of such devices is thus largely dependant on the required stimulation parameters. More recently, closed-loop neuromodulation devices have emerged that intervene when strictly needed. In addition to stimulation, they rely on real-time biopotential sensing, made possible by low noise recording electronics. Recently, FDA-approved/CE-marked, clinically-available examples include the Medtronic Activa $\mathrm{PC}+\mathrm{S}$ system for DBS therapy and NeuroPace Responsive Neurostimulation (RNS) for partial or focal epilepsy. On one hand such closed-loop platforms allow for energy saving by optimising the stimulation delivery function. Nevertheless, the additional circuitry for sensing, data conversion, signal processing and closed-loop algorithms significantly increases the system complexity and thus also the total system power requirements.

The vast majority of implantable systems delivering open loop treatments can operate autonomously - with a single primary cell - for up to 10-15 years before replacement [2]. Closed-loop devices however tend to have higher power requirements and therefore the energy capacity provided by implantable batteries is marginal to provide continuous operation for periods of over a year. For this reason, the AIMD industry has started also using rechargeable battery technology. These allow for a significant increase in implant lifespan, despite having a lower capacity per unit volume. Although this addresses the fundamental energy capacity challenge, it brings in: (i) design challenges, e.g. need for reliable wireless charging techniques and additional power management circuits, and (ii) patient adoption concerns, e.g. device/tissue interface overheating, recharging time and recharging interval. It is thus crucial to assess the expected power requirements, and energy budget needed early in the design process.

\section{Implementation Constraints and Number of Modules}

Typically, AIMDs are limited to single unit devices and implanted either in the upper chest, abdominal area or head. They connect to the neural interface (i.e. electrode) target or stimulation and/or recordingusing one or multiple implantable leads. Emerging multi-module implantable systems propose a distributed approach to the interaction with the body, and partitioning of hardware [5]. These typically have a single,

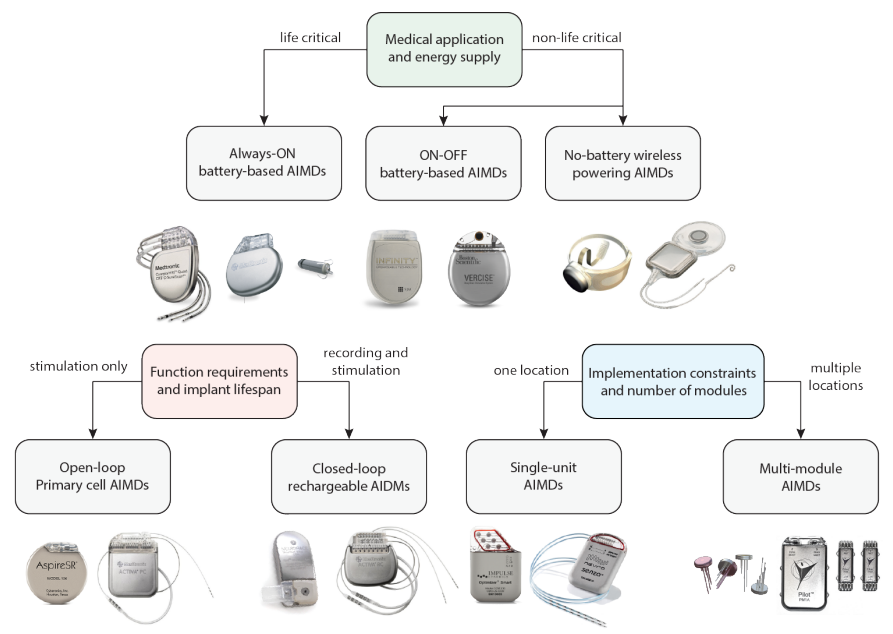

Fig. 1. Key factors driving trends in AIMDs showing current clinical devices.

centralised power source that distributes power to other modules. The decision on which energy generation modality is used (whether implanted battery or external wireless powering) in this case will determine also how the power is distributed from the central device to the peripheral modules.

The most common and reliable method for transferring power within the body is via a wired connection, i.e. with central implant $(\mathrm{CI})$ housing the battery and connecting to the peripheral implants (PIs) through implantable leads [6]. In addition to facilitating power transfer from CI to PIs, these cables can also be used to achieve bidirectional data communication channel, forming a network in which the CI operates as a hub for the PI nodes. Such an example is the networked neuroprosthetic system (NNP) that employs distributed modules, each with dedicated stimulation or recording functions, to interact with the peripheral nervous system for neuroprosthetic applications [3].

The same multi-module trend is observed in next generation implantable neural microsystems that connect to the brain (e.g. brain machine interfaces); allowing reach across wider or multiple brain regions. This can: (i) obtain a better holistic view of the neural activity; and (ii) allow for a multidirectional intervention tool. However, due to biocompatibility, reliability and space constraints such approaches requires a completely wireless solution, posing a new set of challenges [7]. Ongoing research is focused on achieving new wireless means of distributed power delivery within the skull/brain (to multiple devices) using different modalities - electromagnetic, ultrasound, or optical [7]-[9]. An added challenge here, is how to efficiently transmit energy through the skull. Hybrid solutions here deliver wireless power transfer transcutaneously, which in turn employs a wireline link to distribute the power to active subcranial implants [10], [11].

For all different types of AIMDs however, depending on location and number of modules, specific power interfacing circuits are required to transmit (from 'central' implant) and recover power (at 'peripheral' implants).

\section{CAse Study: CANDO Project}

The CANDO project is developing an implantable system to provide an optogenetic treatment for patients suffering from focal Epilepsy [4]. The envisioned therapy relies on a closedloop algorithm to predict and/or detect seizures and intervene in time to suppress them and inhibit them spreading. CANDO 
TABLE I

EXAMPLES OF COMMERCIALLY-AVAILABLE AIMDS COMPARED TO THE CANDO SYSTEM.

\begin{tabular}{|c|c|c|c|c|c|c|}
\hline Parameter & $\begin{array}{c}\text { Med-El } \\
\text { Synchrony }\end{array}$ & $\begin{array}{l}\text { Medtronic } \\
\text { Activa PC }\end{array}$ & $\begin{array}{c}\text { Medtronic } \\
\text { Activa } \mathrm{RC}+\mathrm{S}\end{array}$ & $\begin{array}{l}\text { NeuroPace } \\
\text { RNS }\end{array}$ & $\begin{array}{c}\text { CWRU } \\
\text { NNP System [3] }\end{array}$ & $\begin{array}{c}\text { CANDO } \\
\text { System [4] }\end{array}$ \\
\hline Application & Cochlear Implant & DBS Therapy & DBS Therapy & Epilepsy & Neuroprosthetics & Epilepsy \\
\hline Neuromodulation control & Open-loop & Open-loop & Closed-loop & Closed-loop & Closed loop & Closed-loop \\
\hline Energy source & Wireless powering & Primary cell & Rechargeable & Primary cell & Rechargeable & Rechargeable \\
\hline Battery capacity & $350 \mathrm{mAh}(2$ cells $)$ & $6.3 \mathrm{Ah}$ & $500 \mathrm{mAh}$ & $706 \mathrm{mAh}$ & N/A & $600 \mathrm{mAh}$ \\
\hline Battery lifetime & $60 \mathrm{hr}$ recharge & 4-6 years & 7-14 days/charge & 3-9 years & N/A & $24 \mathrm{hr}$ recharge \\
\hline Location (active device) & Head (skull) & Chest & Chest & Head (skull) & Torso and limbs & Chest and brain \\
\hline Modularity & Single unit & Single unit & Single unit & Single unit & Multi-module & Multi-module \\
\hline
\end{tabular}

implements a closed-loop strategy by sensing neural activity electrically, processing this in real time, and modulating network activity using optogenetic stimulation. The combined electrical/optogenetic neural interface is facilitated using a novel optrode array [4].

\section{A. Power Management Strategy}

By considering the key end-user, system and application requirements as have been previously identified, the overall system power specifications and power management strategy for the CANDO system can be established.

1) Medical Application and Energy Supply: In the target application, seizures may occur at any moment of the day or night. The closed loop strategy continually monitors neural activity and, on detection of key biomarkers, provides realtime intervention through neural stimulation. This requires the implant system to always be on, and therefore the power management strategy includes an implanted battery to ensure a continuous, uninterrupted supply.

2) Functional Requirements and Implant Lifespan: To achieve this real-time intervention requires the front-end acquisition circuits (neural recording amplifiers, filters, data converter), in addition to digital signal processor to continuously operate, thus forming a baseline static power consumption. The stimulation function on the other hand is activated in response to a seizure detection, and thus energy requirements depend on the occurrence frequency of the seizures. Despite this somewhat sporadic nature of the neuromodulation, the power requirement of the light sources used to facilitate the optogenetic activation of neurons is not negligible - it is estimated this require an additional $>10 \mathrm{~mW}$ average power during stimulation. Considering the maximum energy density of a typical implantable battery is $\sim 1 \mathrm{Wh} / \mathrm{cm}^{3}$ [12], a recharge-

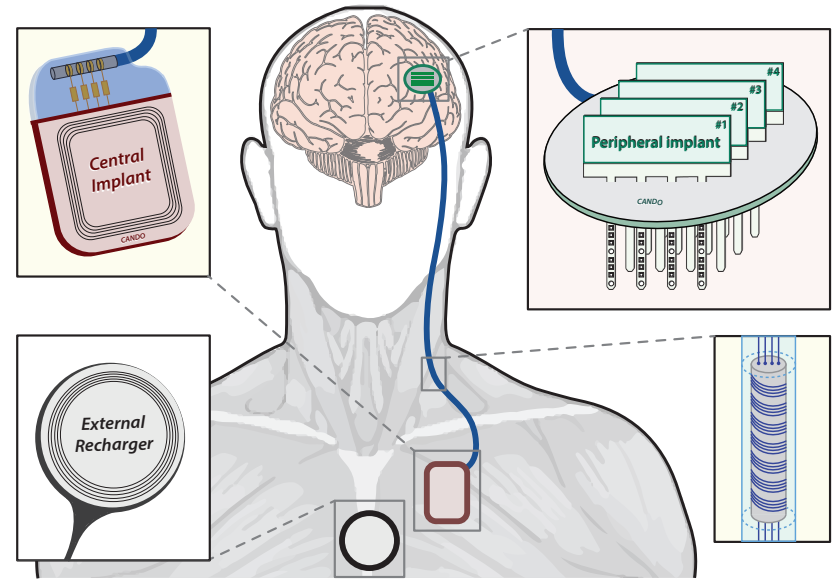

Fig. 2. Concept of the CANDO implant system with (i) an external recharger, (ii) one central implant in the chest, and (iii) multiple peripheral implants at the brain end. able battery is necessary to guarantee an implant lifetime of at least 2 years. This in turn leads to further trade-offs from the end-user and application perspective between battery (i.e. central implant) volume and recharge interval.

3) Implementation Constraints and Number of Modules: Since the implant system needs to directly interface (record and stimulate) at multiple sites near the focal seizure (targeting patients in which this is superficially in the cortex), and given size constraints, the system needs to be partitioned. A multimodule approach is thus taken, allowing for the front-end functions to be performed by 4 Peripheral Implants (PIs) located at different points on the surface of the brain, while confining the high-computation and power consuming processing function to the Central Implant (CI) placed subcutaneously in the chest. This encloses also the rechargeable battery, together with the power management circuits responsible for generating the power supply for the whole implant system. A multiwire implantable lead creates a link between the CI and the PIs to allow for AC power signals to be transferred to the battery-less PIs, which in turn recover the DC supply for their front-end circuitry. The battery is recharged daily with an external portable recharger that couples inductively to the central implant. This system organisation is illustrated in Fig. 2

\section{B. System Implementation}

After defining the end-user/application requirements and system specifications of the CANDO medical device, a power management strategy can be devised to optimise the energy usage and power efficiency, shown in Fig. 3. It is important to start with the hard constraints that are set by the core circuit implementation, e.g. supply requirements for neural recording, stimulation, processing, etc, listed in Table. II.

In designing the power management architecture, there are two key block types that need to be configured: (1) power delivery and recovery blocks (e.g. for wirelessly charging implanted battery, or intrabody power transmission through a wired, AC link); and (2) power conversion blocks (for converting a voltage supply from one level to another, or regulating to a stable level to reduce supply noise).

1) Architecture: The overall system is divided into three main device types: the external charger, central implant (CI) and peripheral implants (PIs). Within the implanted portion,

TABLE II

ESSENTIAL POWER SUPPLY REQUIREMENTS

\begin{tabular}{lll}
\hline Blocks & Voltage domain & Requirement \\
\hline Processing & Digital $(1.8 \mathrm{~V})$ & Stability \\
Recording & Analogue $(1.8 \mathrm{~V})$ & Low noise \\
Stimulation & High voltage $(\geq 4 \mathrm{~V})$ & High efficiency \\
Power management & High voltage $(\geq 4 \mathrm{~V})$ & Varying load and voltage \\
\hline
\end{tabular}




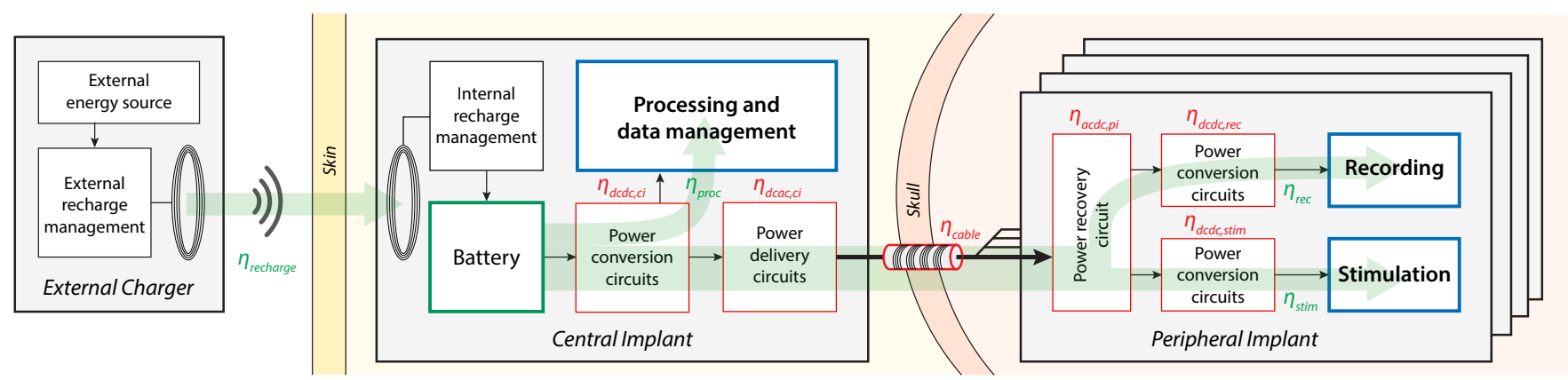

Fig. 3. Power management architecture for the CANDO system showing the overall organisation and power distribution strategy.

the CI power management unit (PMU) needs to perform 3 functions: (1) to facilitate wireless charging, i.e. recover wireless power and recharge the implanted battery $(3.7 \mathrm{~V} \mathrm{Li}$ Ion secondary cell); (2) provide a reduced supply voltage for embedded processor $(1.8 \mathrm{~V})$; and (3) generate an increased supply voltage for AC power transmission to PIs. In turn the PI PMU needs to recover a DC supply from the received $\mathrm{AC}$ carrier and provide two stable DC supplies $(1.8 \mathrm{~V}$ for core/recording circuits and $5 \mathrm{~V}$ for stimulation circuits).

2) Design Considerations: The first design consideration here is the selection of voltage range for power delivery between CI and PIs. With the designated 4-wire power delivery system [5], [10], the power consumed by the connection itself can be estimated by: $P_{4 W}=V_{\text {in }}^{2} C_{\text {para }} f+I_{L}^{2} R$, where $\mathrm{f}$ is the carrier frequency for the AC-coupled 4-wire interface; $\mathrm{I}_{L}$ is the load current. To deliver a specific power budget $\left(\mathrm{V}_{i n} \mathrm{I}_{L}\right)$, there is design trade-off between voltage and current, by taking the cable characteristics into consideration. The input voltage $\left(\mathrm{V}_{i n}\right)$ is constrained by the device voltage rating and subsequent conversion block requirements. For the CANDO system a peak-to-peak AC voltage of $(\approx 7 \mathrm{~V})$ is selected for power transmission. This balances IR cable losses whilst limiting complexity and voltage compliance.

The second design consideration is the selection between linear and switching conversion blocks. For a typical linear conversion the total power consumption within the regulator can be simplified to: $P_{L D O}=V_{i n} I_{d c}+V_{D O} I_{L}$, where $\mathrm{V}_{D O}$ is the dropout voltage across the conversion domains; and $\mathrm{I}_{d c}$ is the static power consumption for the LDO, which is limited by speed and stability requirements. For power conversion blocks bridging voltage domains with large differences, $\mathrm{V}_{D O}$ becomes significant, and introduce excessive power loss in the pass transistors. DC-DC converters here can improve the Power Transfer Efficiency (PTE), even after accounting for switching losses, however this is at the expense of large passive devices, and increased power supply noise. For the CANDO system we have used step-down DC-DC converters in both CI $(3.7 \mathrm{~V}$ to $2.0 \mathrm{~V})$ and PIs $(5 \mathrm{~V}$ to $2.5 \mathrm{~V})$ and cascade with a linear regulator to provide the clean, stable $1.8 \mathrm{~V}$ supply.

3) Evaluation: Determining the overall efficiency and required power budget in a closed-loop AIMD is unfortunately not straight forward - the core system power consumption is highly dependant on stimulation parameters, algorithm complexity and also patient physiology, all of which are variable and largely unknown. The PTE also varies with load current. It is thus helpful to consider the available power budget based on user constraints. Using the CANDO project as a case study, the largest acceptable (from end-user perspective) rechargeable battery has an energy capacity of $600 \mathrm{mAh}$. This must provide continuous therapy over a recharge cycle of approximately $24 \mathrm{hrs}$. Allowing for a $25 \%$ margin, this equates to a $600 \mathrm{mAh} / 30 \mathrm{hrs}=20 \mathrm{~mA}$ average system current consumption. Considering the power management strategy detailed herein, it is estimated (through system simulation) that the PMU within each of the 4 PIs consume approx. $35 \%$ of the total PI power budget. The wireline transmission achieves approximately $80 \%$ efficiency, thus resulting in a best case PTE of 53\% (excluding the CI PMU consumption). It is estimated that $50 \%$ the CI power budget will be allocated to powering the PIs, therefore equating to $3.7 \mathrm{~V} \times 20 \mathrm{~mA} \times 50 \%$ (PI to total power budget) $\times 53 \%$ (overall CI to PI PTE)/4(PI modules $)=4.9 \mathrm{~mW}$ average core power consumption per PI.

\section{CONCLUSION}

The complexity in the design and optimisation of a power management strategy for implantables is increasing with device ambition. The selection of key parameters such as power source, system partitioning, distribution and conversion methods are driven by application requirements, enduser desirability, and system constraints. Through our work on CANDO, we have followed this design methodology in developing a bespoke power management strategy. This has however revealed that for advanced AIMDs, the power budget required for power management can exceed that required for the core device function (i.e. to overall system PTE is $<50 \%$ ).

\section{ACKNOWLEDGEMENT}

The CANDO project is funded by Wellcome Trust/EPSRC.

\section{REFERENCES}

[1] European Standard, "BS EN 45502-1:2015," 2015, BSI, Implants for surgery. Active implantable medical devices.

[2] A. B. Amar et al., "Power approaches for implantable medical devices," sensors, vol. 15, pp. 28 889-28914, 2015.

[3] A. J. Bullard et al., "Design and testing of a 96-channel neural interface module for the networked neuroprosthesis system," Bioelectronic Medicine, vol. 5, no. 1, p. 3, 2019.

[4] R. Ramezani et al., "On-probe neural interface ASIC for combined electrical recording and optogenetic stimulation," IEEE Trans. Biomed. Circuits Syst., vol. 12, no. 3, pp. 576-588, 2018.

[5] A. Mifsud et al., "Adaptive power regulation and data delivery for multimodule implants," in Proc. IEEE BioCAS, 2017, pp. 1-4.

[6] D. Haci et al., "Design considerations for ground referencing in multimodule neural implants," in Proc. IEEE BioCAS, 2018, pp. 1-4.

[7] N. Ahmadi et al., "Towards a distributed, chronically-implantable neural interface," in IEEE/EMBS Conf. on Neural Eng., 2019, pp. 719-724.

[8] D. Seo et al., "Neural dust: An ultrasonic, low power solution for chronic brain-machine interfaces," arXiv preprint arXiv:1307.2196, 2013.

[9] J. S. Ho et al., "Midfield wireless powering for implantable systems," Proceedings of the IEEE, vol. 101, no. 6, pp. 1369-1378, 2013.

[10] S. S. Ghoreishizadeh et al., "Four-wire interface ASIC for a multiimplant link," IEEE Trans. Circuits Syst. I, Reg. Papers, vol. 64, no. 12, pp. 3056-3067, 2017.

[11] E. Musk et al., "An integrated brain-machine interface platform with thousands of channels," bioRxiv, 2019.

[12] D. C. Bock et al., "Batteries used to power implantable biomedical devices," Electrochimica acta, vol. 84, pp. 155-164, 2012. 